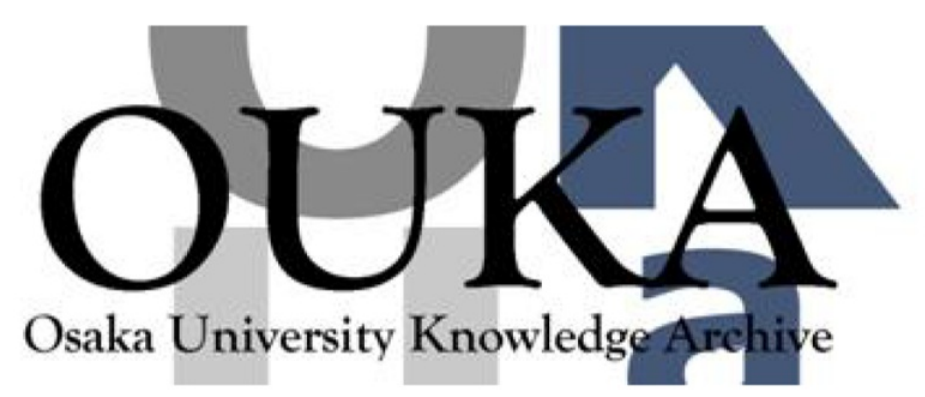

\begin{tabular}{|c|l|}
\hline Title & $\begin{array}{l}\text { Tunable single photonic defect-mode in } \\
\text { cholesteric liquid crystals with laser-induced } \\
\text { local modifications of helix }\end{array}$ \\
\hline Author(s) & $\begin{array}{l}\text { Yoshida, Hiroyuki; Lee, Chee Heng; Fuji i, } \\
\text { Akihiko et al. }\end{array}$ \\
\hline Citation & $\begin{array}{l}\text { Applied Physics Letters. 89(23) p.231913- } \\
\text { p. } 231913\end{array}$ \\
\hline Issue Date & $2006-12-05$ \\
\hline oaire:version & VoR \\
\hline URL & https://hdl.handle. net/11094/75659 \\
\hline rights & \\
\hline Note & \\
\hline
\end{tabular}

Osaka University Knowledge Archive : OUKA

https://ir. Library. osaka-u. ac. jp/

Osaka University 


\section{Tunable single photonic defect-mode in cholesteric liquid crystals with laser-induced local modifications of helix}

Cite as: Appl. Phys. Lett. 89, 231913 (2006); https://doi.org/10.1063/1.2400070

Submitted: 17 August 2006 . Accepted: 21 October 2006. Published Online: 05 December 2006

Hiroyuki Yoshida, Chee Heng Lee, Akihiko Fujii, and Masanori Ozaki

\section{ARTICLES YOU MAY BE INTERESTED IN}

Position sensitive, continuous wavelength tunable laser based on photopolymerizable cholesteric liquid crystals with an in-plane helix alignment

Applied Physics Letters 94, 093306 (2009); https://doi.org/10.1063/1.3089846

Defect mode lasing from a double-layered dye-doped polymeric cholesteric liquid crystal films with a thin rubbed defect layer

Applied Physics Letters 90, 261108 (2007); https://doi.org/10.1063/1.2752017

Electrically color-tunable defect mode lasing in one-dimensional photonic-band-gap system containing liquid crystal

Applied Physics Letters 82, 3593 (2003); https://doi.org/10.1063/1.1577829

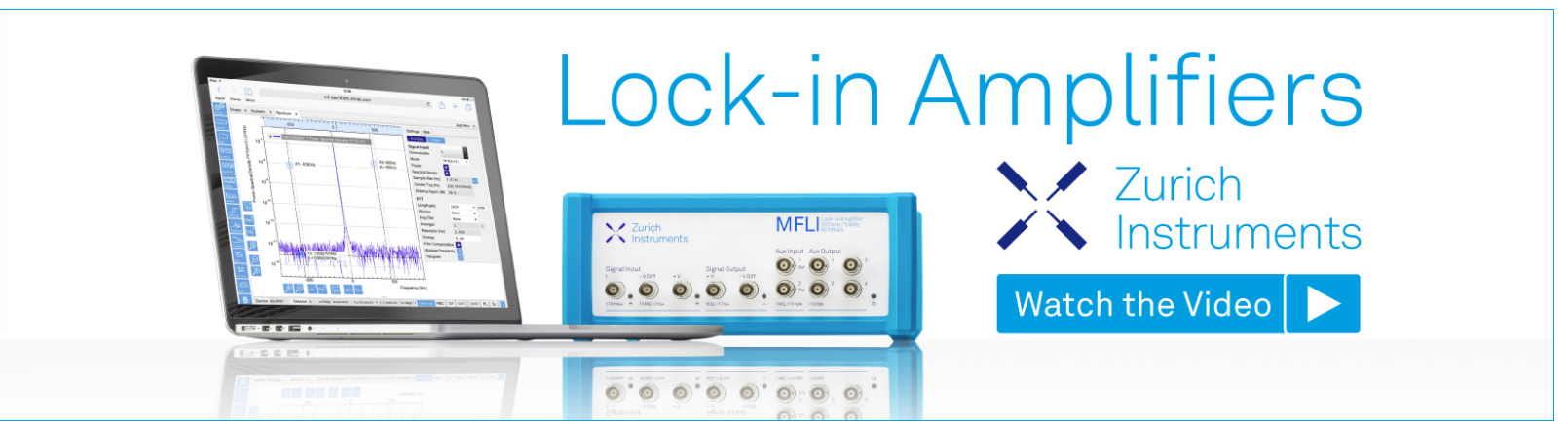




\title{
Tunable single photonic defect-mode in cholesteric liquid crystals with laser-induced local modifications of helix
}

\author{
Hiroyuki Yoshida, ${ }^{\text {a) }}$ Chee Heng Lee, Akihiko Fujii, and Masanori Ozaki ${ }^{\text {b) }}$ \\ Department of Electrical, Electronic and Informational Engineering, Graduate School of Engineering, \\ Osaka University, 2-1 Yamada-oka, Suita, Osaka 565-0871, Japan
}

(Received 17 August 2006; accepted 21 October 2006; published online 5 December 2006)

\begin{abstract}
The authors demonstrate a tunable single photonic defect-mode in a single cholesteric liquid crystal material based on a structural defect introduced by local modification of the helix. An unpolymerized region of cholesteric liquid crystal acting as the defect was left between two polymerized regions via a two-photon excitation laser-lithography process. Upon polymerization, the cholesteric liquid crystal helix elongated and became thermally stable, and a single photonic defect mode was exhibited due to the contrast in the helix pitch at the defect. The defect mode showed tunability upon heating, and a $36 \mathrm{~nm}$ redshift was seen over a temperature range of $30{ }^{\circ}$ C. () 2006 American Institute of Physics. [DOI: 10.1063/1.2400070]
\end{abstract}

Cholesteric liquid crystals (ChLCs) with chiral constituents spontaneously form a helical periodic structure with a periodicity called the pitch. Because of the periodic structure, they exhibit selective reflection (SR), a photonic band gap for circularly polarized light with the same handedness as the material itself. ${ }^{1}$ The photonic properties of these selforganizing, pseudo-one-dimensional photonic band-gap materials have been investigated extensively. Low-threshold laser action has been reported from the SR band edge of $\mathrm{ChLCs},{ }^{2}$ and tuning of the lasing wavelength has been reported, utilizing their ability to change the pitch by external fields such as heat ${ }^{3}$ or optical fields. ${ }^{4}$ On the other hand, laser action with high thermal stability has been reported in photopolymerizable ChLCs (PChLCs) in which the helical structure has been stabilized by polymerization. ${ }^{5}$

Introduction of structural defects in ChLCs has also attracted notable interest. Numerous defect structures had been proposed by stacking PChLC films, due to its simple fabrication method. Low-threshold lasing from the twist defect mode was demonstrated in a stacked configuration of two PChLC films, ${ }^{6,7}$ while hetero-ChLC structures with a nematic LC (Refs. 8 and 9) or a ChLC (Ref. 10) defect layer placed between two PChLC films were reported to show interesting optical properties such as the optical diode effect. In this study, we present a nonstacking process to introduce structural defects in a single PChLC material, and demonstrate a tunable single photonic defect mode, which has not yet been demonstrated by the stacking method.

Figure 1 shows the experimental procedure of introducing the structural defect. The sample cell is prepared by aligning a PChLC material with the helical axis perpendicular to the substrate. We irradiate $100 \mathrm{fs}$ pulses of a Ti:sapphire laser (Spectra Physics: Maitai) at $\lambda=800 \mathrm{~nm}$ and repetition rate of $80 \mathrm{MHz}$ on the sample positioned on a confocal laser scanning microscope (CLSM) system (Zeiss: LSM-510). The laser is focused by a high-numerical aperture (NA) objective lens (oil immersion, 63×, NA of 1.4), and is controlled by a galvanometer to scan a rectangular area of approximately $146.2 \times 146.2 \mu \mathrm{m}^{2}$. In the vicinity of the laser

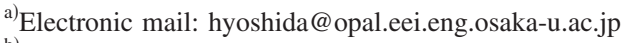

${ }^{b)}$ Electronic mail: ozaki@opal.eei.eng.osaka-u.ac.jp
}

focus where the photon density is high, a two-photon absorption process is induced, polymerizing a finite region within the sample cell [Fig. 1(b)]. The unpolymerized region left between two photopolymerized $\mathrm{ChLC}$ regions formed at both substrates acts as the structural defect [Fig. 1(e)].

The sample used in experiment was prepared by mixing two right-handed PChLC mixtures, 02-595 and 02-596 (Merck) at a ratio of 56:44 (which polymerizes at $\lambda$ $\sim 400 \mathrm{~nm}$ ), after which $1 \mathrm{wt} \%$ of 4-(dicyanomethylene)-2methyl-6-(4-dimethylaminostyryl)-4H-pyran (DCM) (Exci- (a)

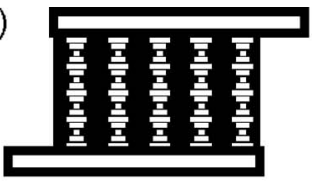

PChLC material planar alignment

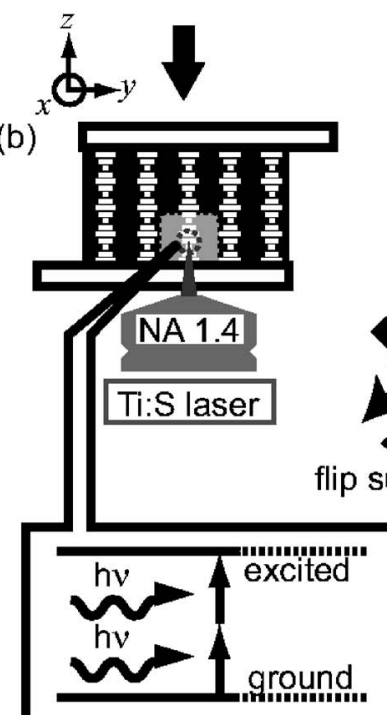

laser-lithography by two-photon polymerization

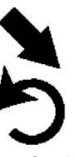

(e)

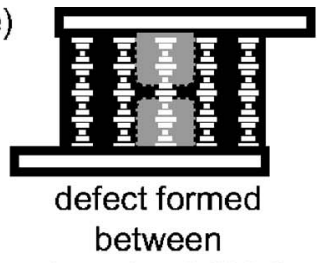

between

polymerized ChLCs

(d)

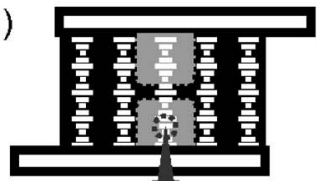

NA 1.4

Ti:S laser

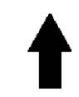

(c)

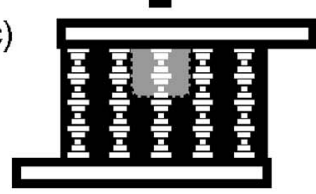

unpolymerized ChLC

polymerized ChLC
FIG. 1. Schematic illustration of the experimental procedure to introduce a structural defect in ChLCs. 


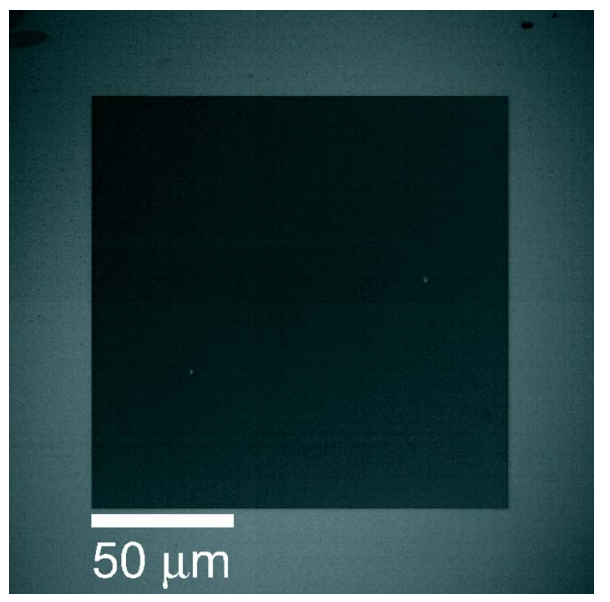

FIG. 2. (Color online) CLSM image of the sample after a laser scan was performed in the square area shown in image. The fluorescence was seen to diminish upon polymerization.

ton) dye was dissolved for observation purposes. The SR band edge is at $\lambda \sim 600 \mathrm{~nm}$, so it can be distinguished from the absorption of the DCM dye. The sample was infiltrated in a cell with cell gap of approximately $5.7 \mu \mathrm{m}$ at a temperature slightly above the clearing point $\left(\sim 106.5^{\circ} \mathrm{C}\right)$ and slowly cooled to $45^{\circ} \mathrm{C}$ where it is in the ChLC phase (laser lithography was also performed at $45^{\circ} \mathrm{C}$ because the sample crystallizes at room temperature). Laser lithography was performed at a laser intensity of $0.61 \mathrm{GW} \mathrm{cm}^{-2}$ and scan speed of $126 \mu \mathrm{m} \mu \mathrm{s}^{-2}$. Only a single scan was required for polymerization: a CLSM image taken after the scan (Fig. 2, taken at $0.1 \mathrm{GW} \mathrm{cm}^{-2}$ ) showed diminished fluorescence of the DCM dye in the polymerized region and could be distinguished from the unpolymerized ChLC. The polymer thickness was approximately $2.5 \mu \mathrm{m}$ yielding an $\sim 0.7 \mu \mathrm{m}$ wide unpolymerized region acting as the defect.

We first characterized the changes induced in the PChLC upon inducing local photopolymerization. One could expect a slight change in the refractive index of the material after polymerization, but to our surprise an anomalous elongation of the helix caused the optical characteristics to change drastically. Figure 3 shows the transmittance spectra of the PChLC before and after single-side laser lithography [corresponding to Figs. 1(a) and 1(c) respectively], for rightcircularly and left-circularly polarized lights, measured on a polarization microscope (Nikon: Eclipse E600 POL) with an objective with NA of 0.4 by a charge-coupled device spectrometer (Hamamatsu Photonics: PMA-11). When a finite ChLC region was polymerized, an extra SR band (shaded in graph) appeared at a longer wavelength region than for the unpolymerized ChLC. Numerical calculations based on the $4 \times 4$ matrix formulation ${ }^{11}$ revealed that the two SR bands corresponded to pitch lengths of 331 and $403 \mathrm{~nm}$ (using $n_{e}$ $=1.72$ and $\left.n_{o}=1.53\right)$, indicating that pitch elongation had occurred. We believe this anomalous elongation of helix is caused by the diffusion of the chiral dopant in the PChLC mixture from the polymerized region into the unpolymerized region for the following reasons: (i) the elongation of the helix occurred only when polymerization was induced locally by laser lithography (not when the whole material was polymerized uniformly by UV light), indicating that a finite polymerized region needs to be surrounded by an unpolymerized region for pitch elongation to occur, (ii) the dimin-
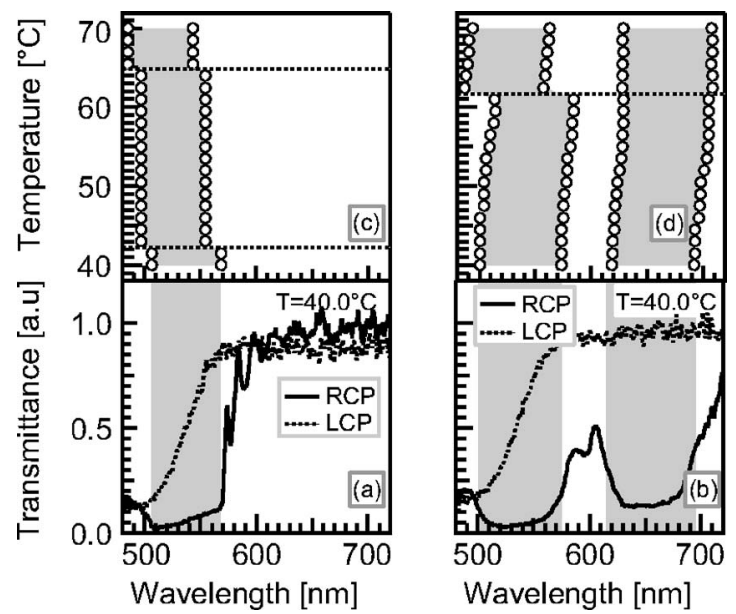

FIG. 3. Transmittance spectra at $40{ }^{\circ} \mathrm{C}$ and the temperature dependence of the SR bands (shaded region) of the PChLC material [(a) and (c)] before laser lithography [Fig. 1(a)] and [(b) and (d)] after a polymerized region was formed on a single substrate [Fig. 1(c)]. The absorption of the DCM dye is observed at $<550 \mathrm{~nm}$.

ished DCM fluorescence from the polymerized region indicates that certain nonpolymeric components such as the DCM dye and chiral dopant in the PChLC mixture may have come out of the polymer matrix after polymerization, and (iii) the polymer slowly changed color after laser lithography, indicating a gradual process. Because there is a contrast in the pitch length in the polymerized (bulk) and unpolymerized (defect) regions, we can expect a defect mode to appear in the SR band. ${ }^{12}$

The temperature dependence of the SR bands [Figs. 3(c) and $3(d)$ ] indicates higher heat stability in the polymerized ChLC. In the unpolymerized ChLC, a stepwise blueshift of the SR band is observed by heating, corresponding to a discrete shortening of the pitch due to a increase in the number of half pitches existing in the liquid crystal cell. ${ }^{3}$ On the other hand, the SR band of the polymerized ChLC does not show a discrete pitch shortening by heating, indicating that the helix became stable against the external heat. The stabilization is a result of the dense cross-linking of the monomers upon polymerization, which prevents the molecules to move freely to either wind or unwind. ${ }^{5}$ A tunable photonic defect mode should utilize this difference in the thermal response of the two regions. The gradual redshift observed in the SR bands in Fig. 3(d) may be due to the expansion of the polymer matrix upon heating, and the gradual unwinding of the ChLC at the polymer-LC interface with weaker anchoring. This is still under investigation along with the actual LC director distribution at the newly formed interface, but is contrary to the dynamics in Fig. 3(c) where there is strong anchoring and no gradual shift of the SR band is observed.

Figure 4(a) shows the transmittance spectrum of the structure with polymerized ChLC regions at both substrate surfaces of the sample cell [Fig. 1(e)]. A single defect mode is exhibited at $\lambda=645 \mathrm{~nm}$ within the SR band of the elongated helix of the polymerized ChLC, exhibited between 605 and $695 \mathrm{~nm}$. Although the distribution of the LC director in this structure with newly formed surfaces still needs characterization and should be discussed elsewhere, a theoretical calculation of the transmittance spectrum using parameters described above gives good agreement with the experimental results, supporting that a single photonic defect mode was 


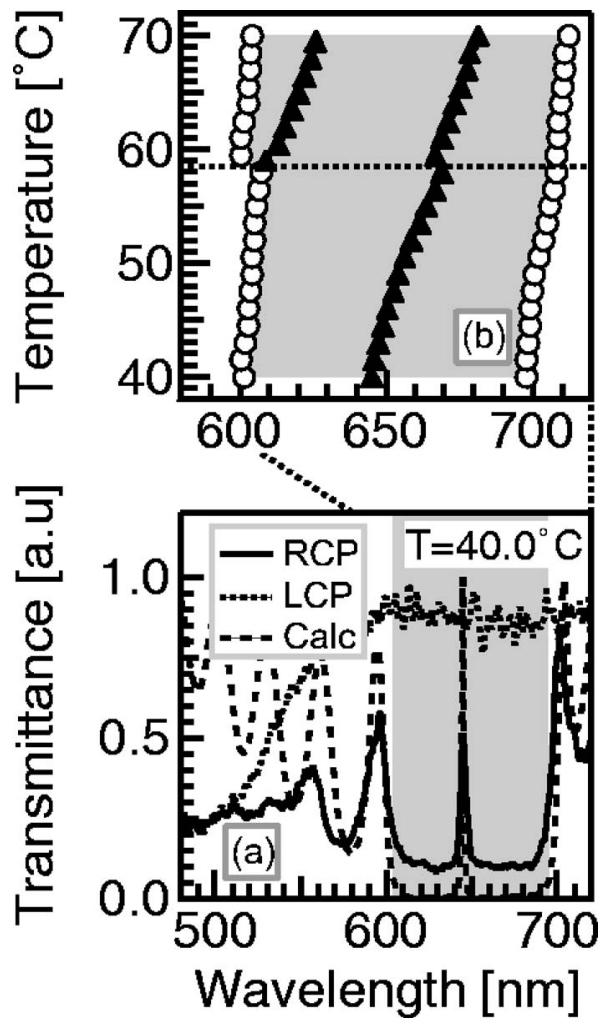

FIG. 4. (a) Transmittance spectra at $40{ }^{\circ} \mathrm{C}$ and (b) temperature dependence of the defect mode wavelength and the SR band (shaded gray) of the PChLC material after forming polymerized regions on both substrates [Fig. 1(e)].

realized from the unpolymerized $\mathrm{ChLC}$ acting as a defect introduced between two polymerized ChLC regions.

The tunability of the defect mode was demonstrated by heating the sample from 40 to $70{ }^{\circ} \mathrm{C}$ [Fig. 4(b)]. The defect mode wavelength redshifted upon heating, exhibiting a $36 \mathrm{~nm}$ shift over the $30^{\circ} \mathrm{C}$ temperature range. The main cause of this shift may be the expansion of the polymer, diminishing the width of the defect. A different stepwise shift of the defect mode was observed at $59.0^{\circ} \mathrm{C}$, corresponding to a discrete shortening of the ChLC pitch at the defect in contrast to the bulk material. This may be caused by the $\mathrm{ChLC}$ at the defect favoring the pitch-shortened state at high temperature because of the surrounding ChLC with a discretely shortened pitch, although at the defect the unpolymerized ChLC has two polymer-LC interfaces with "weaker" anchoring. The increase in the contrast of the two pitch lengths is also accountable for the second defect mode appearing in the SR band. Therefore, a tunable single photonic defect mode is achieved in a single PChLC material because of the laser-induced helix modulation.

In conclusion, we demonstrated a tunable single photonic defect mode in ChLCs based on inducing local photopolymerization by two-photon excitation laser lithography. The ChLC pitch elongated by a factor of about $20 \%$ and became thermally stable after laser lithography, thus creating a contrast in the pitch between the polymerized and the unpolymerized regions, enabling the structure act as a defect. We fabricated a structure with two polymerized layers sandwiching an unpolymerized region approximately $\sim 0.7 \mu \mathrm{m}$ wide, in which a single defect mode was realized. The defect mode wavelength was variable by $36 \mathrm{~nm}$ upon heating the sample by $30{ }^{\circ} \mathrm{C}$ and causing an expansion of the polymer as well as a shortening of the $\mathrm{ChLC}$ pitch in the unpolymerized region. These optical devices may be used as tunable narrow bandpass filters or heat sensors in integrated optical circuits.

The authors thank Merck KGaA for the PChLC materials. This work is partly supported by a Grant-in-Aid for Scientific Research from the Ministry of Education, Culture, Sports, Science and Technology of Japan.

${ }^{1}$ H. deVries, Acta Crystallogr. 4, 219 (1951).

${ }^{2}$ V. I. Kopp, B. Fan, H. K. M. Vithana, and A. Z. Genack, Opt. Lett. 23, 1707 (1998).

${ }^{3}$ K. Funamoto, M. Ozaki, and K. Yoshino, Jpn. J. Appl. Phys., Part 2 42, L1523 (2003).

${ }^{4}$ P. V. Shibaev, R. L. Sanford, D. Chiappetta, V. Milner, A. Genack, and A. Bobrovsky, Opt. Lett. 13, 2358 (2005).

${ }^{5}$ T. Matsui, R. Ozaki, K. Funamoto, M. Ozaki, and K. Yoshino, Appl. Phys. Lett. 81, 3741 (2002).

${ }^{6}$ M. Ozaki, R. Ozaki, T. Matsui, and K. Yoshino, Jpn. J. Appl. Phys., Part 1 42, 472 (2003).

${ }^{7}$ J. Schmidke, W. Stille, and H. Finkelmann, Phys. Rev. Lett. 90, 083902 (2003).

${ }^{8}$ J. Hwang, M.-H. Song, B.-C. Park, S. Nishimura, T. Toyooka, J. W. Wu, Y. Takanishi, K. Ishikawa, and H. Takezoe, Nat. Mater. 4, 383 (2005).

${ }^{9}$ M.-H. Song, B. Park, K.-C. Shin, T. Ohta, Y. Tsunoda, H. Hoshi, Y. Takanishi, K. Ishikawa, J. Watanabe, S. Nishimura, T. Toyooka, Z. Zhu, T. M. Swager, and H. Takezoe, Adv. Mater. (Weinheim, Ger.) 16, 779 (2004).

${ }^{10}$ M.-H. Song, N. Y. Ha, K. Amemiya, B. Park, Y. Takanishi, K. Ishikawa, J. W. Wu, S. Nishimura, T. Toyooka, and H. Takezoe, Adv. Mater. (Weinheim, Ger.) 18, 193 (2006).

${ }^{11}$ D. W. Berreman, J. Opt. Soc. Am. 62, 502 (1972).

${ }^{12}$ T. Matsui, M. Ozaki, and K. Yoshino, Phys. Rev. E 69, 061715 (2004). 